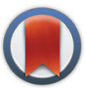

CrossMark \&lick for updates

Cite this: Polym. Chem., 2016, 7, 878

\title{
Micelles from self-assembled double-hydrophilic PHEMA-glycopolymer-diblock copolymers as multivalent scaffolds for lectin binding $\dagger$
}

\author{
H. Park,,$^{\mathrm{a}}$ S. Walta, $\neq^{\mathrm{b}}$ R. R. Rosencrantz,,$^{\mathrm{c}}$ A. Körner, ${ }^{\mathrm{a}}$ C. Schulte, ${ }^{\mathrm{d}}$ L. Elling, ${ }^{\star c}$ \\ W. Richtering*b and A. Böker* $\S^{a}$
}

We introduce a novel double-hydrophilic hydroxyethylmethacrylate (HEMA) based diblock glycopolymer which self-assembles into homogeneous spherical micellar structures in water. The micellar structure renders surface-oriented $\mathrm{N}$-acetylglucocosamine (GlcNAc) sugar moieties for strong multivalent glycanmediated lectin binding. Structural analysis and lectin binding is performed by microscopy methods, dynamic light scattering (DLS) and two-focus fluorescence correlation spectroscopy (2fFCS), revealing a novel micellar type of multivalent sugar binding scaffold with high potential for biomedical applications.

Received 26th May 2015,

Accepted 30th November 2015

DOI: $10.1039 / c 5 p y 00797 f$

www.rsc.org/polymers controlled glycan-ligand presentation. Due to the hydrophilicity of sugar moieties caused by their large number of hydroxyl groups, polymerisable neo-glycoconjugates have been used to synthesise amphiphilic block glycopolymers by conjugation to a hydrophobic polymer block for applications like emulsion stabilisation, drug delivery or for lectin binding studies. ${ }^{7-9}$ These amphiphilic diblock glycopolymers can self-assemble into various architectures such as micelles or vesicles. Their spherical and three dimensional structures may display much higher affinity towards binding lectins due to their larger surface area and therefore possible multivalency. ${ }^{10,11}$

Lectins are generally known as carbohydrate binding proteins with considerable specificity for certain glycans. ${ }^{12,13}$ Plant lectins are often used in diagnostic assays and glycan analysis of glycoproteins. ${ }^{14-18}$ In human glycan-binding proteins have a huge impact in biomedical research. ${ }^{19-21}$ Lectins are known to play important roles in tumor progression and tumor angiogenesis depending solely on the cell type and its glycosylation pattern, the cell glyco-code. ${ }^{22-25}$ Furthermore, bacterial toxins are known to bind to their host cell via lectin domains as initial step of their pathogenity. ${ }^{26,27}$ Lectins are therefore therapeutic and diagnostic targets which can be addressed by their specific carbohydrate ligands.

Glycan presentation in close proximity to each other enhances binding strength by orders of magnitude and is therefore the key element of effective glycan-functionalized materials in biomedical applications. ${ }^{28-30}$ Recently, glycovesicles have been shown to increase the affinity of a model lectin (Con A) about 40 times due to the multivalent presentation. ${ }^{31}$ Moreover, a drastic increase in binding ability was shown with sequence variation of hetero multivalent glycopolymers again in the case of ConA. ${ }^{32}$ Giving a good hint, that the general 
approach of using glycopolymers as scaffolds for multivalent presentation is certainly highly promising. ConA, however, is an example of a tetrameric lectin bearing both adjacent and opposite binding sites. But increased avidity towards multivalent scaffolds may not be found for all lectins just by increasing the number of presented ligands. ${ }^{33}$ This highlights the urge of tailored materials to produce high-affinity ligands in glycoresearch, especially as there are various structures of lectins known. ${ }^{34}$ Nevertheless, targeted drug release or tissue imaging may be realised by utilising glycan-lectin interactions as steering wheel, leading the containers to their destination. ${ }^{35-37}$ Such containers need to bear a more hydrophobic interior for interaction with the imaging dye or drug and a sugar coat for targeting and strong binding. Encouragingly it has been reported that amphiphilic mucin-mimiking glycopolymers are readily incorporated into cell membranes and undergo intracellular trafficking. ${ }^{38}$ However, the formation of micelles and therefore the action as container of these structures is unlikely, because of the size relations between large hydrophilic glycopolymer and small hydrophobic endgroups.

So far, less research has been directed towards the synthesis of double-hydrophilic glycopolymers, which are capable of micelle formation, owing to the synthetic difficulty. Most micellar or vesicular approaches combined glycans or glycopolymers with small aliphatic molecules to form multivalent glycovesicles. ${ }^{39-41}$ However, very good binding of lectins to these have been shown. Recently, we fabricated multivalent glycopolymer brushes on silicon wafers by surface-initiated atom transfer radical polymerisation (SI-ATRP) with additional biocatalytic elongations of glycans directly on the silicon wafer substrate. ${ }^{42}$ This system does not only show high multivalency but also enables the simultaneous binding of adjacent as well as opposing binding sites. Neighbouring binding sites may interact with two glycans on the same brush, whereas opposite sites bind two brushes by crosslinking. Both effects are maintained by flexibility of the brushes on the one hand and high grafting density on the other.

In this work, ATRP was applied for the synthesis of welldefined self-assembling glycopolymer micelles. The micelles should maintain the same good binding abilities as shown for surface grafted brushes due to low distance between two glycans in all dimensions. Interestingly, the micelles were formed although the diblock copolymer consists of two rather hydrophilic blocks. The self-assembly of double-hydrophilic block copolymers by slight differences in overall hydrophilicity of the blocks was recently reported. ${ }^{43}$ However, no biological application has been proven so far. Our structures were analysed by various methods such as TEM and DLS. The critical micelle concentration (CMC) was determined by incorporation of small hydrophobic molecules. Additionally, we investigated the polarity and container properties of the self-assembled aggregates by incorporation of the polarity-sensitive fluorescent dye Nile Red. Confocal laser scanning microscopy and fluorescence spectroscopy were carried out with the dyed micelles. Finally, lectin binding to the micelles and determination of the binding strength was carried out by 2 fFCS.

\section{Experimental section}

\section{Synthesis of diblock glycopolymer}

The diblock glycopolymer consists of one block of poly(2hydroxyethyl methacrylate) (PHEMA) and a block of poly(2-O( $N$-acetyl- $\beta$-D-glucosamine)ethyl methacrylate) (PGlcNAcEMA) (Fig. 1). For the preparation of diblock glycopolymer PHEMA- $b$ PGlcNAcEMA (4), firstly PHEMA (2) was synthesised by ATRP starting from HEMA (1). A representative polymerisation reaction is as followed: $15.0 \mathrm{~mL}(123.50 \mathrm{mmol})$ HEMA was placed into the reaction flask and stirred in $15.0 \mathrm{~mL}$ of methyl ethyl ketone $($ MEK) $/ 1$-propanol $=7 / 3(\mathrm{v} / \mathrm{v})$. The reaction mixture was degassed with nitrogen and preheated to $50{ }^{\circ} \mathrm{C}$ for $20 \mathrm{~min}$. Then, $354.32 \mathrm{mg}(2.47 \mathrm{mmol}) \mathrm{CuBr}, 0.77 \mathrm{~g}$ (4.94 mmol) bpy and $362.5 \mu \mathrm{L}(2.47 \mathrm{mmol})$ ethyl $\alpha$-bromoisobutyrate were added and stirred under a nitrogen stream until homogeneous dark brown solution is formed. The reactor was degassed with nitrogen for further $20 \mathrm{~min}$. The polymerisation proceeded at $50{ }^{\circ} \mathrm{C}$ for $24 \mathrm{~h}$. The second block based on a polymerisable $\mathrm{N}$-acetyl-glucosamine (GlcNAc) derivative (GlcNAcEMA, 3) was then added utilising ATRP again, now with the PHEMA-block as macroinitiator. $1.08 \mathrm{~g}(3.25 \mathrm{mmol})$ of the glycomonomer was stirred in $10 \mathrm{~mL}$ methanol/water $=1 / 1(\mathrm{v} / \mathrm{v})$ until the complete dissolution and deoxygenated by nitrogen purging. $5.15 \mathrm{mg}(0.052 \mathrm{mmol}) \mathrm{CuBr}, 2.90 \mathrm{mg}(0.013 \mathrm{mmol}) \mathrm{CuBr}_{2}$, $20.30 \mathrm{mg} \quad(0.13 \mathrm{mg}) \quad 2,2^{\prime}$-bipyridine and $291.64 \mathrm{mg}$ $(0.065 \mathrm{mmol})$ macroinitiator were added and stirred under a nitrogen stream for $20 \mathrm{~min}$. The reaction was performed at

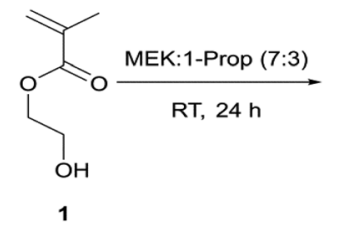

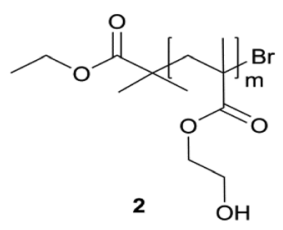

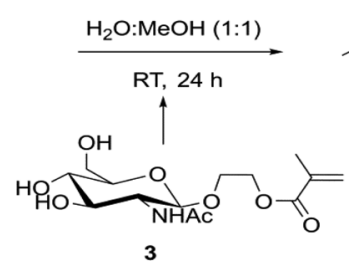

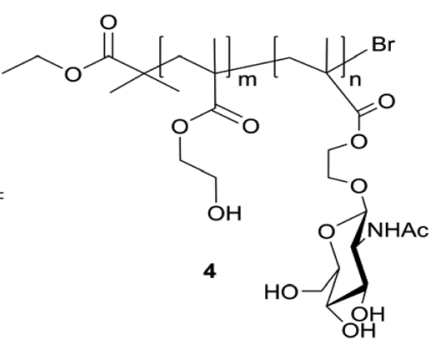

Fig. 1 General scheme for the synthesis of PHEMA- $b$-PGIcNAcEMA. 
room temperature for $15 \mathrm{~h}$. The purified polymer was freezedried and a white polymer was obtained.

The synthesised polymers were characterised by ${ }^{1} \mathrm{H}-\mathrm{NMR}$, GPC and MALDI-ToF MS (see ESI $\dagger$ ). The conversion of HEMA to PHEMA was determined to be $90 \%$ resulting in a theoretical degree of polymerisation (DP) of 45. For the PGlcNAcEMAblock a theoretical DP of approx. 10 was calculated. According to MALDI-TOF MS analysis the diblock glycopolymer shows an average DP of approx. 39 for the HEMA unit and a DP of 4 for the GlcNAcEMA unit. An average molecular weight $M_{\mathrm{n}}$ of approx. $6546 \mathrm{~g} \mathrm{~mol}^{-1}$ and a low polydispersity $M_{\mathrm{W}} / M_{\mathrm{n}}$ of 1.1, indicating a uniform size of the polymers, was determined for PHEMA- $b$-PGIcNAcEMA.

\section{Protein treatment}

Lectins GS-II were purchased from Vector Laboratories (Burlingame, CA, US) as FITC conjugates. As residual unbound dye was interfering with the measurements, it was purified via size exclusion chromatography (SEC) utilising Sephadex® G-25 with LB (with $10 \mathrm{mM}$ HEPES-NaOH, pH = 7.5, $150 \mathrm{mM} \mathrm{NaCl}$, $0.1 \mathrm{mM} \mathrm{CaCl}_{2}$ ) to give a protein concentration of $20 \mu \mathrm{gml}^{-1}$.

\section{CMC determination}

For CMC determination $5 \mathrm{mg}$ benzoylacetone (Sigma-Aldrich, Steinheim, Germany) was dissolved in $500 \mathrm{~mL}$ bidest water. Different concentrations of PHEMA- $b$-PGlcNAcEMA were dissolved in $100 \mu \mathrm{L}$ of this solution and the absorption spectra were recorded utilising a microtiterplate reader. The CMC is determined as the concentration where the absorption band at $315 \mathrm{~nm}$ reaches its minimum.

\section{Viscosity determination}

The dynamic viscosity is an important parameter in the Stokes-Einstein equation, in order to obtain the hydrodynamic radius from the diffusion coefficient. The dynamic viscosity was calculated by the equation $\eta=\nu \cdot \rho$, whereas the density was determined with a DMA-5000 density meter (Anton-Paar, Germany). The kinematic viscosity was measured with a Lauda PVS viscosity system. For this purpose a Micro-Ostwald capillary (No. 1065402, capillary constant $0.0106 \mathrm{~mm}^{2} \mathrm{~s}^{-2}$ ) was used. Thus, a dynamic viscosity of $0.9195 \pm 0.0009 \mathrm{mPa}$ s was obtained for aqueous buffer solution (LB) used in DLS and $2 \mathrm{fFCS}$ measurements. The temperature was set to $25^{\circ} \mathrm{C}$.

\section{Dynamic light scattering (DLS)}

DLS measurements were performed on a standard ALV setup equipped with HeNe laser (JDS Uniphase, $633 \mathrm{~nm}, 35 \mathrm{~mW}$ ), digital hardware correlator (ALV 5000), two avalanche photo diodes (Perkin Elmer, SPCM-CD2969), goniometer (ALV, CGS-8F) and light scattering electronics (ALV, LSE-5003). Highly diluted samples were prepared to prevent multiple scattering. Before measurements, the samples were filtered several times through regenerated cellulose filters (Sartorius) with a pore size of $0.2 \mu \mathrm{m}$. Measurements were recorded in pseudocross correlation mode. The scattering angle was varied between $30^{\circ}$ and $142^{\circ}$ in steps of $4^{\circ}$ for PHEMA- $b$-PGIcNAcEMA and between $34^{\circ}$ and $124^{\circ}$ in steps of $3^{\circ}$ for GS-II. Measurement times were $90 \mathrm{~s}$ and $240 \mathrm{~s}$, respectively. The temperature was set to $25{ }^{\circ} \mathrm{C}$. Data were evaluated in the following way: intensity autocorrelation functions were transformed to electric field autocorrelation functions by use of the Siegert relation. A second order cumulant analysis was applied to obtain the decay rate $\Gamma_{2}$. The cumulant fit range was limited between the lag time of $10 \mu$ s and the lag time where the electric field autocorrelation function amplitude had decayed to 30 percent of the experimental maximum value. $\Gamma_{2}$ was plotted against the square of the scattering vector $q$, the mean diffusion coefficient $\bar{D}$ was obtained from the equation

$$
\Gamma_{2}=\bar{D} q^{2}
$$

The hydrodynamic radius $R_{\mathrm{h}}$ was calculated by use of the Stokes-Einstein equation.

\section{Two-focus fluorescence correlation spectroscopy (2fFCS)}

2fFCS measurements were performed on a time-resolved confocal fluorescence microscopy system (PicoQuant, MicroTime200 with dual-focus option). For excitation, two perpendicular polarised pulsed laser beams (wavelengths of $470 \mathrm{~nm}$ or $637 \mathrm{~nm}$ were used) are combined with a broadband polarising beamsplitter cube (Ealing Catalog) and then coupled into a polarisation maintaining single mode fiber. The laser pulses are generated by two identical laser diode heads (Picoquant, LDH-P-C-470 or LDH-P-C-635B) with an overall repetition rate of $40 \mathrm{MHz}$ and thus a temporal distance of $25 \mathrm{~ns}$ between successive pulses. The synchronisation of the laser pulses (so-called pulsed interleaved excitation ${ }^{44}$ ) with alternating orthogonal polarisation is achieved by laser driver electronics (PicoQuant, PDL 828 "Sepia II"). At the fiber output the light is collimated by a lens and afterwards reflected by a dichroic mirror (AHF-Analysentechnik, z470/532/637rpc) towards the water-immersion objective (Olympus, UPLSAPO $60 x W, 1.2$ N.A.). Prior to the dichroic mirror, the total laser power was adjusted to approx. $25 \mu \mathrm{W}$. In front of the objective a Nomarski prism (Olympus, U-DICTHC) is located, which deflects the incoming laser pulses in two different directions according to their corresponding polarisation. After focusing the light through the objective of the inverted microscope (Olympus, IX71), two overlapping but laterally shifted foci are generated in the sample. The particle diffusion is only dominated by the time a particle needs to diffuse from the first focus to the second focus, so it only depends on the distance between the focal volumes but not their exact size and shape. Because this interfocal distance remains constant over time for a given wavelength, it enables us to measure absolute values of the diffusion coefficient. ${ }^{45}$ The fluorescence light is collected by the same objective, passed through the prism and the dichroic mirror. It is then focused onto a confocal pinhole of $75 \mu \mathrm{m}$ diameter to block out-of-focus light and achieve a good signal-to-noise ratio. After additional collimation, the light passes through laser clean up filters (AHF-Analysentechnik, HQ505/30m for $470 \mathrm{~nm}$, HQ687/70m 
$637 \mathrm{~nm})$. Both beam contributions are guided by a nonpolarising beam splitter cube onto the active area of two single photon avalanche diodes (Micro Photon Devices, PDM series). A dedicated time-correlated single photon counting (TCSPC) ${ }^{46}$ electronics (PicoQuant, PicoHarp 300) is used to refer every detected photon to its arrival time from the start of the experiment and with respect to the last laser pulse. It is known for every recorded photon which laser pulse has excited it and thus in which focus the photon was generated. Autocorrelation functions (ACFs) for each focus and cross correlation functions (CCFs) between the two foci are calculated from the photon arrival time traces with a specific algorithm based on a multiple-tau approach, ${ }^{47}$ but only for photons from different detectors to avoid after pulsing artifacts. ${ }^{48}$ A custom-made Matlab routine was used for computation.

For subsequent data fitting it has to be taken into account that the fluorescence intensity depends on the detection efficiency and the shape of the molecular detection function (MDF) in both foci. In 2fFCS the MDF is described by a twoparameter model based on a modified three-dimensional elliptic Gaussian distribution: ${ }^{45}$

$$
U(\vec{r})=\frac{\kappa(z)}{w^{2}(z)} \exp \left[-\frac{2}{w^{2}(z)}\left(x^{2}+y^{2}\right)\right]
$$

with the beam waist function

$$
w(z)=w_{0}\left[1+\left(\frac{\lambda_{\mathrm{ex}} z}{\pi w_{0}^{2} n}\right)^{2}\right]^{1 / 2}
$$

and amplitude function

$$
\kappa(z)=1-\exp \left(-\frac{2 a^{2}}{R^{2}(z)}\right),
$$

where $R(z)$ is given by

$$
R(z)=R_{0}\left[1+\left(\frac{\lambda_{\mathrm{em}} z}{\pi R_{0}^{2} n}\right)^{2}\right]^{1 / 2} .
$$

In the above equations $\{x, y, z\}$ are Cartesian coordinates with $z$ along the optical axis, $\lambda_{\text {ex }}$ refers to the excitation wavelength, $\lambda_{\text {em }}$ to the center emission wavelength, $n$ is the refractive index of the immersion medium (water), $a$ denotes the radius of the confocal pinhole divided by magnification, and $w_{0}$ and $R_{0}$ are two initially unknown model parameters that are determined by the fit. Taking together the parameterisation of the MDF for each focus and the solution of the diffusion equation (Green function), the following function is used to fit the ACFs and CCFs:

$$
\begin{aligned}
g(\tau, \delta)= & g_{\infty}(\delta)+\frac{\varepsilon_{1} \varepsilon_{2} c}{4} \sqrt{\frac{\pi}{D \tau}} \int_{-\infty}^{+\infty} \mathrm{d} z_{1} \int_{-\infty}^{+\infty} \mathrm{d} z_{2} \frac{\kappa\left(z_{1}\right) \kappa\left(z_{2}\right)}{8 D \tau+w^{2}\left(z_{1}\right)+w^{2}\left(z_{2}\right)} \\
& \times \exp \left[-\frac{\left(z_{2}-z_{1}\right)^{2}}{4 D \tau}-\frac{2 \delta^{2}}{8 D \tau+w^{2}\left(z_{1}\right)+w^{2}\left(z_{2}\right)}\right]
\end{aligned}
$$

where $\tau$ is the correlation or lag time, $\delta$ the interfocal distance, $D$ the diffusion coefficient, $c$ the concentration of particles and $\varepsilon_{1}$ and $\varepsilon_{2}$ denote the overall detection efficiencies from both focal volumes. This model curve can be applied to the measured ACFs $\left(\delta=0, \varepsilon_{1} \varepsilon_{2}\right.$ replaced by either $\varepsilon_{1}{ }^{2}$ or $\left.\varepsilon_{2}{ }^{2}\right)$ and CCFs simultaneously in a global fit with a linear least-square fitting approach including, among others, $\delta$ and $D$ as fit parameters. By measuring fluorescent solutions with known diffusion coefficient $\delta$ can be calculated. ${ }^{49}$ In this work, interfocal distances of $\delta=259 \mathrm{~nm}$ (for $\lambda_{\mathrm{ex}}=470 \mathrm{~nm}$ ) and $\delta=$ $287 \mathrm{~nm}$ (for $\lambda_{\mathrm{ex}}=637 \mathrm{~nm}$ ) were determined. The model curve can be extended by additional terms to account for two fluorescent components or triplet blinking.

In this work, measurements took place in sealed sample cells to prevent solvent evaporation and to make use of homemade temperature regulation. ${ }^{50}$ The sample temperature was set to $25{ }^{\circ} \mathrm{C}$ with an absolute accuracy of $\pm 0.1{ }^{\circ} \mathrm{C}$. LiChrosolv (Merck, No. 115333), clean water for chromatography, was used for sample preparation. The measurement time was chosen in such a way to detect around $4 \times 10^{6}$ photons per measuring point. The collected photons were divided into $2 \times$ $10^{6}$ photon packages, thus two packages per point and overall 20 at least (10 packages for inhibition measurements at least). The resulting ACFs and CCFs (two of each for every package) were averaged and fitted by a two-component model in the case of GS-II and a one-component model including triplet blinking in the case of BSA.

\section{Fluorescence spectroscopy}

A $0.8 \mathrm{mM}$ Nile Red stock solution was prepared in ethanol and diluted 1000-fold in the diblock glycopolymer system. Fluorescence spectra were obtained at room temperature using a Jasco FP-6500 Spectrofluorometer under the following conditions: excitation wavelength at $595 \mathrm{~nm}$ and fluorescent emission range between 610 and $750 \mathrm{~nm}, 3 \mathrm{~nm}$ slit width for excitation and emission, resolution $0.1 \mathrm{~nm}$ and a scan speed of $60 \mathrm{~nm} \min ^{-1}$.

\section{Microscopy}

Confocal laser scanning fluorescence microscopy is performed on a Leica TCS SP8 to visualize fluorescent micelles. For this purpose, we used the same Nile Red concentration in the micellar diblock glycopolymer solution as with fluorescence spectroscopy measurements.

TEM images were obtained using a Zeiss Libra ${ }^{\mathrm{TM}} 120$ Microscope. The accelerating voltage of the electron beam was set at $120 \mathrm{kV}$. A drop of the sample was trickled on a carbon-coated copper grid. Then, the copper grid was air-dried under ambient conditions.

In situ TEM experiments were performed at a Zeiss Libra 200FE apparatus equipped with in-column energy filter and Köhler illumination at $200 \mathrm{kV}$. An about $100 \mathrm{eV}$ energy window at the most probable loss of the water plasmon was utilised for image generation. The sample was prepared by pipetting a $0.5 \mu \mathrm{L}$ droplet between two Si3N4-membranes, which were in a Hummingbird scientific in situ sample holder. To reduce contamination artifacts and improve the wettability of the otherwise hydrophobic membranes, an air-plasma was applied for 
$10 \mathrm{~s}$. The water-plasmon in the EELS-spectrum was repeatedly captured during imaging to prove the presence of a liquid film in the sample. Furthermore, occasional diffusion of particles into or out of the focus plane was taken as proof for the presence of the solvent. Magnification was from 16000 to 25000 times.

\section{MALDI-ToF MS analysis}

The PHEMA- $b$-PGlcNAcEMA sample was dissolved in bidest water to a concentration of $5 \mathrm{mg} \mathrm{ml}^{-1}$ and the DHB matrix to a concentration of $20 \mathrm{mg} \mathrm{ml}^{-1}$ in TA30; sample and matrix solution were mixed in a ratio of $1: 1$. The cationisation solution of $0.1 \mathrm{~mol} \mathrm{l}^{-1}$ sodium trifluoracetate in TA 30 was prepared as a thin-layer on the target plate (ground steel target, Bruker) and $1 \mu \mathrm{l}$ of the sample/matrix solution added on top of it. Mass spectra were acquired using a $1 \mathrm{kHz}$ Laser Bruker UTX MALDIToF mass spectrometer (Bruker, Bremen, Germany) with pulsed ion extraction. The spectra were recorded in the reflector mode with a repetition rate of $1000 \mathrm{~Hz}$ signals from positive ions; 3000 spectra were added to a sum spectrum. While recording the sample stage moved in a random fashion on a sample spot allowing 500 laser shots per position. The calibration was carried out externally using a mixture of proteins (ProteinCalibrationStandard I, \#206375 from Bruker Daltonics, Bremen, Germany) prepared on a near neighbour spot. The spectra were baseline subtracted (Top hat algorithm) and mass peak annotation was obtained with the centroid detection algorithm (width 0.5 at $80 \%$ height).

The characterisation of the copolymer molecular weight distributions, determination of polydispersity and mixture deconvolution were performed with the Polytools ${ }^{\mathrm{TM}}$ software (Bruker Daltonics, Bremen) and the Polymerix ${ }^{\mathrm{TM}}$ software tool from Sierra Analytics, Modesto, CA 95356 USA.

\section{Results and discussion}

The diblock glycopolymer was dissolved in water at a molar concentration of $3.2 \times 10^{-4} \mathrm{~mol} \mathrm{~L}^{-1}$ for electron microscopy analysis, Fig. 2a-c presents the micrographic images of PHEMA- $b$-PGlcNAcEMA. Uniformly distributed dark spherical structures are obtained by TEM measurements (Fig. 2a and b) with sizes around $21 \mathrm{~nm}$ in radius. In addition, about $5 \%$ of the particles occur as large aggregates, which can be also observed, as shown in the in situ TEM micrograph (c). However, the smaller micellar structures are the dominating species and monodisperse in size distribution.

Actually, the synthesised block copolymers are composed of two hydrophilic blocks: one water soluble glycooligomer consisting of PHEMA conjugated to a very hydrophilic sugar and a water-soluble pure PHEMA block. It has been reported that PHEMA homopolymers with a DP of 39, which corresponds to our PHEMA block (see ESI $\dagger$ ), are considered as water-soluble, but possess a cloud point of at least $32^{\circ} \mathrm{C}$. As all experiments were carried out at room temperature, the PHEMA block can be considered fully soluble. ${ }^{51}$ Despite the existence of two hydrophilic blocks, these block glycopolymers form spherical particle structures in water. We assume that these structures have a micellar structure with the more hydrophilic glycan moieties at the outside. Other studies so far found a hydrophilic PHEMA corona or at least outer core in blockcopolymers micelles at elevated temperatures. ${ }^{51}$

We determined the critical micelle concentration (CMC) by analysing the absorption spectra of benzoylacetone (BZA) at different concentrations of blockcopolymer (see ESI, Fig. S10 ). BZA is known to exhibit two absorption bands; one at about $250 \mathrm{~nm}$, the other at about $315 \mathrm{~nm}$ due to the existence of ketonic- and enolic forms, respectively. In hydrophobic environments the enolic form is more pronounced, whereas in aqueous solution the ketonic form and therefore the band at $250 \mathrm{~nm}$ is more present. For this, BZA is utilised as marker molecule for CMC determinations, as the environment inside a micelle changes the hydrophilicity in comparison to the surrounding media. ${ }^{52-54}$ Interestingly, our measurements showed that at high concentrations of the block copolymer the band at $315 \mathrm{~nm}$ was more pronounced indeed, whereas at very low concentrations the band at $250 \mathrm{~nm}$ was dominating. In this way we were not only able to determine a CMC of approx. $0.4 \mathrm{~g} \mathrm{~L}^{-1}$ $\left(0.06 \mathrm{mmol} \mathrm{L}^{-1}\right)$, but also to get a hint that the interior of the structure is more hydrophobic than the surrounding media indeed.

These results indicate that the glycans are localised on the outside and the PHEMA block forms the interior. However, the band at $315 \mathrm{~nm}$, even at high concentrations of $\mathbf{4}$, is not as pro-
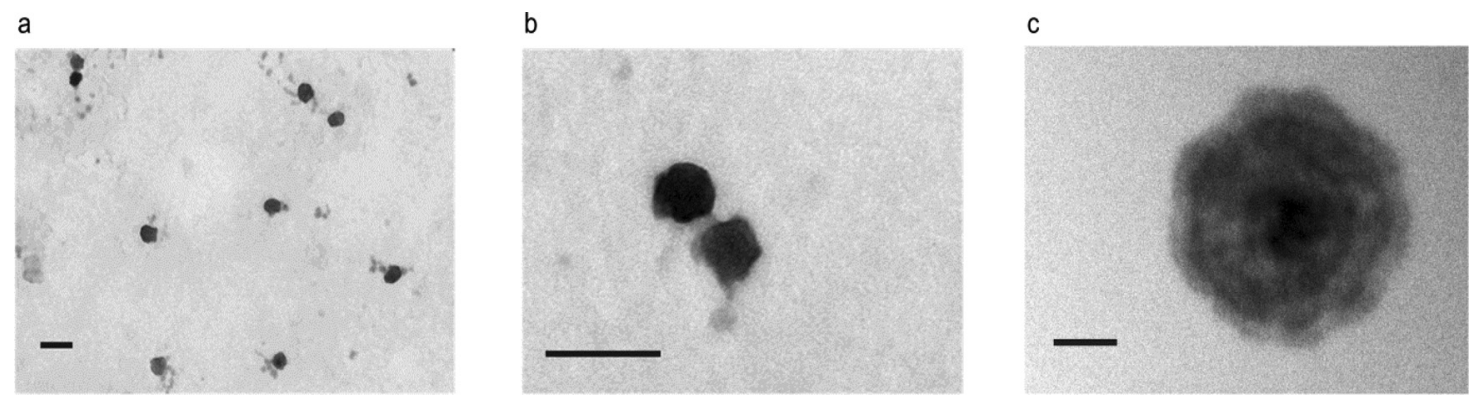

Fig. 2 Micrographs of PHEMA-b-PGIcNAcEMA. (a) and (b) are obtained by TEM. (c) By in situ TEM. The scale bars represent $100 \mathrm{~nm}$. 
nounced as with classical surfactant systems. This may be caused by the water-soluble and still hydrophilic properties of PHEMA, which gives an excellent opportunity of incorporation of small hydrophobic molecules and usage of our system as molecular container.

To probe the abilities of our system to act as a transporter for, e.g., imaging purposes, we tried to incorporate the fluorescent dye Nile Red. It was shown that the fluorescence of Nile Red is environment-sensitive and especially enhanced in a hydrophobic environment. ${ }^{55-57}$ We found a dominant increase in the fluorescence intensity as well as a spectral blue shift of the emission wavelength in our diblock glycopolymer solution compared to the fluorescence in pure water. Both is caused by exposition of Nile Red to a more hydrophobic environment provided by the interior of the micelles and only occurs above the CMC. As shown in Fig. 3a the emission wavelength decreases by $10 \mathrm{~nm}$. Nile Red was used previously as highly polarity-sensitive fluorescent probe to determine hydrophobicity of protein surfaces or polymer films. ${ }^{56-58}$ Therefore we expect to gain more insight about the assembly and polarity of the aggregates. Polarity is often related to mixtures of water and organic solvents. ${ }^{57}$ Taking that into account a decrease of $10 \mathrm{~nm}$ in emission maximum equals the polarity of a binary mixture containing $50 \%$ methanol in water. ${ }^{58}$ This corresponds to a surprisingly high change in relative permittivity, considering the low concentration of polymer in solution. Hence, this change may only be explained by incorporation of the dye molecules into a more hydrophobic nano-environment, with high local concentration of residues with lower relative permittivity, as expected for the micellar structure we suggest. Furthermore the blue shift of emission wavelength also confirms that the interior of the micelles is not as hydrophobic as observed for different polymers and nonpolar organic solvents, where the spectral shift is more pronounced. ${ }^{56-58}$

Moreover, we could image an overview of the micelles in water using confocal laser scanning fluorescence microscopy. Again we detect some large aggregates (Fig. 3b), but also visualise nanosized entities with red fluorescence (Fig. 3c). We conclude that the polymers although combining rather hydrophilic blocks are not only able to self-assemble into micellar structures in aqueous environment, but also may serve as nano-containers for small hydrophobic molecules. The phenomena of controlled self-assembly of double-hydrophilic block copolymers in water has been mostly overlooked so far, thus proven and studied for poly(ethyleneoxide)-poly(2-methyl2-oxazoline) diblock copolymers. ${ }^{43}$

However, the usage of PHEMA as less hydrophilic block in our case gives the opportunity to use these copolymers in biomedical applications due to the biocompatibility of PHEMA. Furthermore, the glycopolymer block bears a high biological coding capacity for tissue or cell targeting. Hence, we wanted to elucidate, if the glycans are accessible for lectin binding. If binding is detected, this will confirm our idea of less hydrophilic PHEMA forming the inside of the micelles and PGEMA forming the hydrophilic corona. That may lead to the possibility of glycan-targeted dye or drug transport.

More detailed analysis of the structures especially with respect to biological interaction was carried out via DLS and $2 \mathrm{fFCS}$ measurements. As the micelles are presenting GlcNAc we choose lectin II from Griffonia simplicifolia (GS-II) as GlcNAc specific lectin to specify the binding in a biological context. GS-II is known as a homodimer with four carbohydrate recognition domains (CRD) and has a $M_{\mathrm{W}}$ of $120 \mathrm{kDa}^{59,60}$

DLS was used as complementary technique to get more precise information about the particle sizes of diblock glycopolymer and lectin. GS-II was dissolved in aqueous buffer solution (LB: $10 \mathrm{mM}$ HEPES-NaOH, $\mathrm{pH}=7.5,150 \mathrm{mM} \mathrm{NaCl}$, $0.1 \mathrm{mM} \mathrm{CaCl}_{2}$ ) to keep the lectin structure stabilised and provide $\mathrm{Ca}^{2+}$-ions for binding. A molar concentration of $9 \mu \mathrm{mol} \mathrm{L}{ }^{-1}$ was used. PHEMA- $b$-PGlcNAcEMA was dissolved in the same buffer solution with a molar concentration of $0.5 \mathrm{mmol} \mathrm{L}^{-1}$, thus above the CMC. Both solutions were filtered through a $0.2 \mu \mathrm{m}$ filter. Fig. $4 \mathrm{a}$ presents the decay rate obtained from the second order cumulant fit plotted against the square of the scattering vector $\mathrm{q}$ for PHEMA- $b$ PGlcNAcEMA (squares) and GS-II (circles). The diffusion a

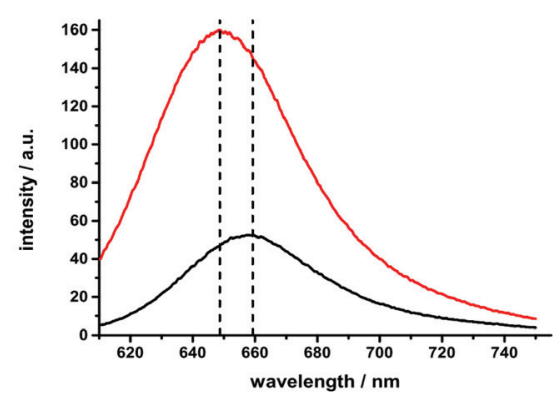

b

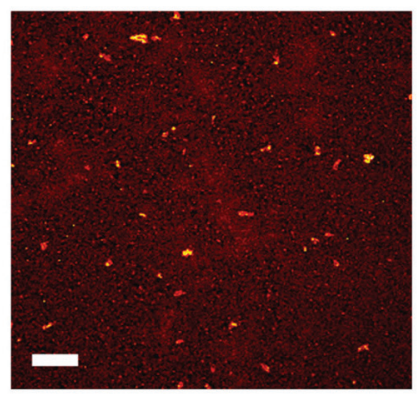

C

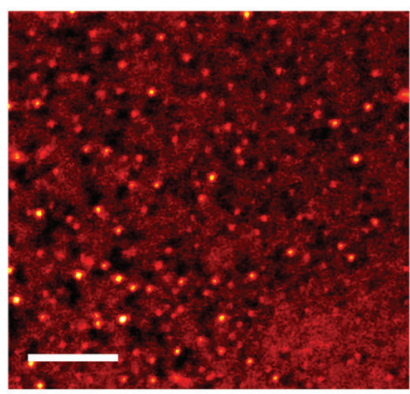

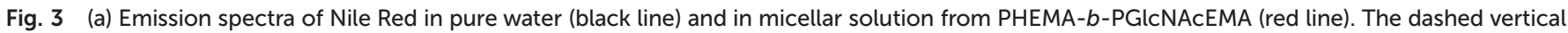

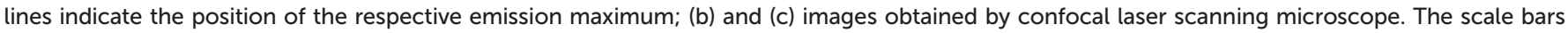
represent $5 \mu \mathrm{m}$. 
a)

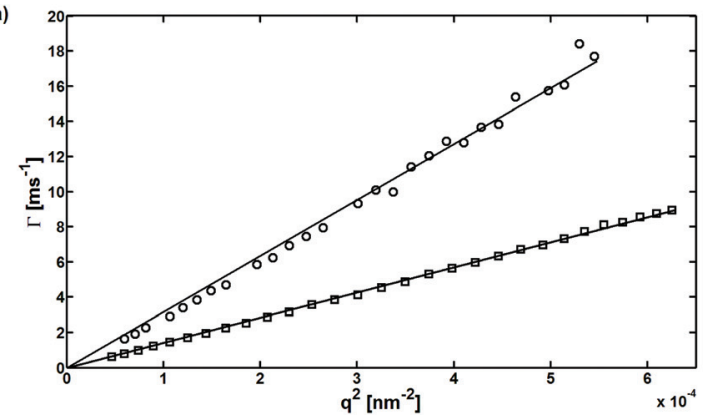

b)

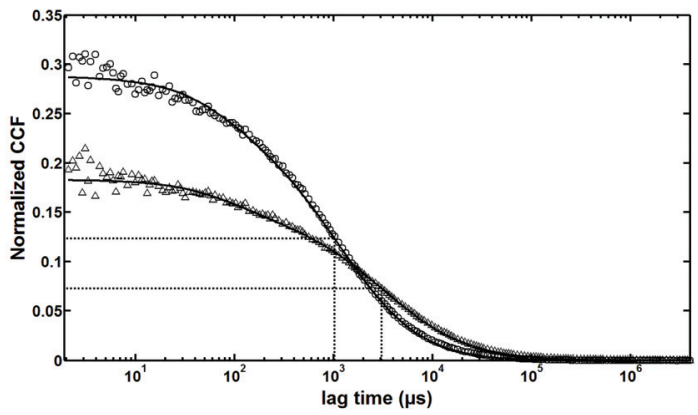

Fig. 4 (a) $2^{\text {nd }}$ order decay rate with $q^{2}$ for GS-II (circles) and PHEMA-b-PGlcNAcEMA (squares). Linear regressions are indicated by solid lines. (b) Normalised cross correlation functions with lag time for GS-II (circles) and GS-II upon addition of PHEMA-b-PGlcNAcEMA (triangles). Two-component fits are indicated by solid curves. The dashed lines display the particular inflection points.

coefficients were calculated from the slope of linear regressions shown as solid lines in Fig. 4a. The good linearisation of PHEMA- $b$-PGlcNAcEMA data indicates a relatively narrow size distribution of micellar structures in solution. ${ }^{61} \mathrm{~A}$ hydrodynamic radius of $16.7 \pm 0.1 \mathrm{~nm}$ was calculated, which verifies the observations made by microscopy. For GS-II a hydrodynamic radius of $7.5 \pm 0.1 \mathrm{~nm}$ was obtained.

Fluorescence correlation spectroscopy (FCS) is well-suited to monitor binding processes by measuring changes in diffusion characteristics. ${ }^{32,62}$ However, standard FCS measurements are affected by different properties of the optical setup, like refractive index mismatch, and the photophysics of the used fluorophores, so that it is rather difficult to obtain absolute values of the diffusion coefficient. These problems can be neglected using $2 \mathrm{fFCS}$ as an improved version of the standard confocal FCS setup. ${ }^{45,49}$ In this work, the protein binding was analysed using FITC labeled GS-II at a molar concentration of $17 \mathrm{nmol} \mathrm{\textrm {L } ^ { - 1 }}$. Unlabeled block glycopolymer was used at a molar concentration of $0.25 \mathrm{mmol} \mathrm{L}^{-1}$. Complete diagrams with auto and cross correlation functions and their fits, for GS-II and PHEMA- $b$-PGlcNAcEMA, respectively, are presented in the ESI (Fig. S11 and S12†). Cross correlation functions (CCFs) were fitted using a two-component fit model of diffusion (Fig. 4b, solid curves), which accounts for the presence of residual FITC (not chemically attached to GS-II). Indeed, the GS-II stock solution was purified by SEC (see ESI, Fig. S9†), but a certain fraction of $30 \%$ of free dye still remained.

Fig. 4b shows the normalised and averaged CCFs plotted against the lag time of freely diffusing GS-II (circles) and GS-II upon addition of PHEMA- $b$-PGlcNAcEMA (triangles). The dashed lines in Fig. 4b indicate that the inflection points of the CCFs are significantly different. This corresponds to a change in diffusion time meaning that, after addition of PHEMA- $b$-PGlcNAcEMA, GS-II was bound to the diblock glycopolymer. The hydrodynamic radii obtained from the fits are in good agreement with DLS and TEM analysis: $6.8 \pm 0.1 \mathrm{~nm}$ for GS-II and $15.4 \pm 0.2 \mathrm{~nm}$ for GS-II upon addition of PHEMA- $b$ PGlcNAcEMA. The slightly larger radius provided by TEM may be caused by flattening of the soft micelles on the target. We conclude that little or no unbound GS-II remained in solution after addition of diblock glycopolymer, which hints to strong multivalent glycan-mediated protein adsorption hence.

To get more information about the binding properties of our glycopolymer we performed 2fFCS measurements under inhibiting conditions for GS-II binding by varying concentrations of the free sugar ligand GlcNAc (Fig. 5). Displacement experiments were carried out by adding GlcNAc after GS-II has been incubated together with the micelles and the binding events are assumed to be completed. Competitive inhibition in contrast describes the simultaneous addition of GS-II and GlcNAc to the micelles. Therefore, in the latter approach the lectin "decides" which presentation mode of GlcNAc is more favoured: dissolved free GlcNAc or multivalent micelle. From these experiments $\mathrm{IC}_{50}$ values, describing the concentration of inhibitor, where $50 \%$ of maximum binding is left, are obtained. Meanwhile GS-II and PHEMA- $b$-PGlcNAcEMA concentrations remained unchanged. The inhibition experiments gave $\mathrm{IC}_{50}$ values of $1.627 \pm 0.3950 \mathrm{mM}$ and $1.049 \pm 0.2613 \mathrm{mM}$ for displacement and competitive inhibitions, respectively.

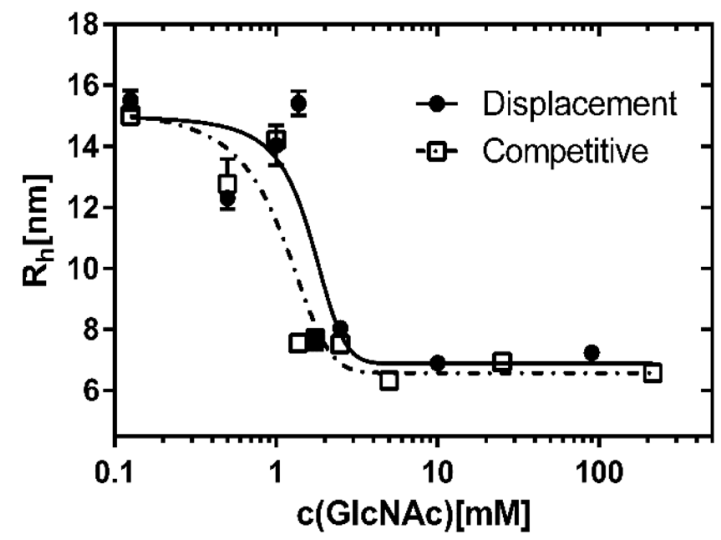

Fig. 5 Inhibiting the binding of lectin GS-II to the micelles with

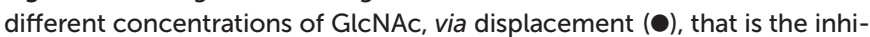
bition of binding by adding GlcNAc after incubation of micelles and GS-II as well as competition ( $\square$ ), which is the addition of GlcNAc and GS-II simultaneously to the micelles. 
These values are surprisingly high in comparison with the inhibition of hemagglutination activity of GS-II by soluble GlcNAc, which is about $0.048 \mathrm{mM}$ for complete inhibition. ${ }^{63}$ This supports our assumption that the glycans are on the outside of the micelles and accessible with high multivalency of the glycopolymer which assures outstanding binding capabilities towards lectins. Because these binding capabilities could also be rest on unspecific binding, further experiments were done by substituting GS-II with bovine serum albumin (BSA) labeled with Alexa Fluor 647. BSA is not known to have any affinity to glycan moieties. The measurements showed that the diffusion coefficient of BSA did not change upon addition of PHEMA-b-PGlcNAcEMA (see ESI, Fig. S13 and S14†). This result supports that our synthesised diblock glycopolymer acts as highly specific ligand for GS-II.

\section{Conclusions}

Herein, PHEMA- $b$-PGlcNAcEMA diblock glycopolymer was synthesised and its structure in water was observed using microscopy measurements such as TEM and in situ TEM. Although the synthesised polymer is composed of two rather hydrophilic parts, the resulted double-hydrophilic diblock glycopolymers have shown to self-assemble into micellar particle structures in aqueous systems. The measured size of micellar particles was around $16 \mathrm{~nm}$ in radius by DLS and 2fFCS. TEM showed slightly larger values due to flattening on the target. The lectin binding ability of the micelles was observed by $2 \mathrm{fFCS}$, showing excellent specific binding of lectin GS-II with very high $\mathrm{IC}_{50}$ values for the inhibition of binding. Moreover, we proved the incorporation of small hydrophobic hardly water-soluble molecules leading to the conclusion that the micelles may serve as transporter. Comparison of the emission spectra of the polarity-sensitive fluorescent probe Nile Red in different reported systems showed a surprisingly high shift in relative permittivity. This proves the formation of micelles as this shift may be explained by a high local concentration of hydrophobic molecules as it is found in micelles. Although double-hydrophilic, the micelles are capable of solubilisation and incorporation of small hydrophobic molecules. Hence, our system may be elaborated to act as nano-container for drugs or dyes with specific targeting by multivalent and strong glycan-protein interactions.

\section{Acknowledgements}

R. R. R., L. E. and A. B. acknowledge funding by the Deutsche Forschungsgemeinschaft (DFG) in the framework of the SFB 985. We thank Dennis Go (DWI - Leibniz Institute for Interactive Materials) for performing confocal laser scanning microscopy and Jan Timper (GFE RWTH Aachen) for in situ TEM imaging.

\section{References}

1 C. Boyer and T. P. Davis, Chem. Commun., 2009, 60296031.

2 S. Pearson, W. Scarano and M. H. Stenzel, Chem. Commun., 2012, 48, 4695-4697.

3 S. Cecioni, A. Imberty and S. Vidal, Chem. Rev., 2015, 115, 525-561.

4 Y. Nagasaki, K. Yasugi, Y. Yamamoto, A. Harada and K. Kataoka, Biomacromolecules, 2001, 2, 1067-1070.

5 Z. Hu, X. Fan and G. Zhang, Carbohydr. Polym., 2010, 79, 119-124.

6 R. Ouhib, B. Renault, H. Mouaziz, C. Nouvel, E. Dellacherie and J.-L. Six, Carbohydr. Polym., 2009, 77, 32-40.

7 F. Suriano, R. Pratt, J. P. K. Tan, N. Wiradharma, A. Nelson, Y.-Y. Yang, P. Dubois and J. L. Hedrick, Biomaterials, 2010, 31, 2637-2645.

8 M. Alvárez-Paino, R. Juan-Rodríguez, R. Cuervo-Rodríguez, A. Muñoz-Bonilla and M. Fernández-García, J. Colloid Interface Sci., 2014, 417, 336-345.

9 O. Otman, P. Boullanger, E. Drockenmuller and T. Hamaide, Beilstein J. Org. Chem., 2010, 6, 58.

10 O. León, A. Muñoz-Bonilla, V. Bordegé, M. Sánchez-Chaves and M. Fernández-García, J. Polym. Sci., Part A: Polym. Chem., 2011, 49, 2627-2635.

11 S. R. S. Ting, G. Chen and M. H. Stenzel, Polym. Chem., 2010, 1, 1392-1412.

12 N. Sharon and H. Lis, Lectins, Kluwer Academic Publishers, 2003.

13 I. E. Liener, N. Sharon and I. J. Goldstein, The Lectins: properties, functions, and applications in biology and medicine, Academic Press, 1986.

14 P. Gemeiner, D. Mislovičová, J. Tkáč, J. Švitel, V. Pätoprstý, E. Hrabárová, G. Kogan and T. Kožár, Biotechnol. Adv., 2009, 27, 1-15.

15 D. Mislovičová, P. Gemeiner, A. Kozarova and T. Kožár, Biologia, 2009, 64, 1-19.

16 Z. Dai, J. Zhou, S. J. Qiu, Y. K. Liu and J. Fan, Electrophoresis, 2009, 30, 2957-2966.

17 Z. Yang and W. S. Hancock, J. Chromatogr., A, 2004, 1053, 79-88.

18 K. Pilobello and L. Mahal, in Microchip-Based Assay Systems, ed. P. Floriano, Humana Press, 2007, vol. 385, pp. 193-203.

19 E. M. Van Damme, in Lectins, ed. J. Hirabayashi, Springer, New York, 2014, vol. 1200, pp. 3-13.

20 S. André, H. Kaltner, J. Manning, P. Murphy and H.-J. Gabius, Molecules, 2015, 20, 1788-1823.

21 C. Bah, E. Fang and T. Ng, in Antitumor Potential and other Emerging Medicinal Properties of Natural Compounds, ed. E. F. Fang and T. B. Ng, Springer, Netherlands, 2013, pp. 55-74.

22 H. Ghazarian, B. Idoni and S. B. Oppenheimer, Acta Histochem., 2011, 113, 236-247.

23 A. H. Ebrahim, Z. Alalawi, L. Mirandola, R. Rakhshanda, S. Dahlbeck, D. Nguyen, M. Jenkins, F. Grizzi, E. Cobos, 
J. A. Figueroa and M. Chiriva-Internati, Ann. Transl. Med., 2014, 2, 88.

24 T. Funasaka, A. Raz and P. Nangia-Makker, Glycobiology, 2014, 24, 886-891.

25 A. W. Griffioen and V. L. Thijssen, Ann. Transl. Med., 2014, 2.

26 H. Genth, S. C. Dreger, J. Huelsenbeck and I. Just, Int. J. Biochem. Cell Biol., 2008, 40, 592-597.

27 B. E. Collins and J. C. Paulson, Curr. Opin. Chem. Biol., 2004, 8, 617-625.

28 M. Monsigny, R. Mayer and A. C. Roche, Carbohydr. Lett., 2000, 4, 35-52.

29 T. K. Dam, R. Roy, S. K. Das, S. Oscarson and C. F. Brewer, J. Biol. Chem., 2000, 275, 14223-14230.

30 N. Parera Pera, H. M. Branderhorst, R. Kooij, C. Maierhofer, M. van der Kaaden, R. M. J. Liskamp, V. Wittmann, R. Ruijtenbeek and R. J. Pieters, ChemBioChem, 2010, 11, 1896-1904.

31 E. Mahon, T. Aastrup and M. Barboiu, Chem. Commun., 2010, 46, 2441-2443.

32 D. Ponader, P. Maffre, J. Aretz, D. Pussak, N. M. Ninnemann, S. Schmidt, P. H. Seeberger, C. Rademacher, G. U. Nienhaus and L. Hartmann, J. Am. Chem. Soc., 2014, 136, 2008-2016.

33 S. R. Stowell, M. Dias-Baruffi, L. Penttila, O. Renkonen, A. K. Nyame and R. D. Cummings, Glycobiology, 2004, 14, 157-167.

34 J. M. Rini, Annu. Rev. Biophys. Biomol. Struct., 1995, 24, 551-577.

35 T. Minko, Adv. Drug Delivery Rev., 2004, 56, 491-509.

36 C. Bies, C.-M. Lehr and J. F. Woodley, Adv. Drug Delivery Rev., 2004, 56, 425-435.

37 M. M. Fuster and J. D. Esko, Nat. Rev. Cancer, 2005, 5, 526542 .

38 D. Rabuka, M. B. Forstner, J. T. Groves and C. R. Bertozzi, J. Am. Chem. Soc., 2008, 130, 5947-5953.

39 Z. Mouline, E. Mahon, E. Gomez, V. Barragan-Montero, J.-L. Montero and M. Barboiu, Chem. Commun., 2014, 50, 731-733.

40 N. Jayaraman, K. Maiti and K. Naresh, Chem. Soc. Rev., 2013, 42, 4640-4656.

41 J. Park, L. H. Rader, G. B. Thomas, E. J. Danoff, D. S. English and P. DeShong, Soft Matter, 2008, 4, 19161921.

42 H. Park, R. R. Rosencrantz, L. Elling and A. Böker, Macromol. Rapid Commun., 2015, 36, 45-54.
43 O. Casse, A. Shkilnyy, J. Linders, C. Mayer, D. Häussinger, A. Völkel, A. F. Thünemann, R. Dimova, H. Cölfen, W. Meier, H. Schlaad and A. Taubert, Macromolecules, 2012, 45, 4772-4777.

44 B. K. Muller, E. Zaychikov, C. Brauchle and D. C. Lamb, Biophys. J., 2005, 89, 3508-3522.

45 T. Dertinger, V. Pacheco, I. von der Hocht, R. Hartmann, I. Gregor and J. Enderlein, ChemPhysChem, 2007, 8, 433443.

46 D. V. O'Connor and D. Phillips, Time-correlated single photon counting, Academic Press, London, 1984, p. 288.

47 M. Wahl, I. Gregor, M. Patting and J. Enderlein, Opt. Express, 2003, 11, 3583-3591.

48 J. Enderlein and I. Gregor, Rev. Sci. Instrum., 2005, 76, 033102.

49 C. B. Müller, A. Loman, V. Pacheco, F. Koberling, D. Willbold, W. Richtering and J. Enderlein, Europhys. Lett., 2008, 83, 5 .

50 C. B. Müller and W. Richtering, Colloid Polym. Sci., 2008, 286, 1215-1222.

51 J. V. M. Weaver, I. Bannister, K. L. Robinson, X. BoriesAzeau, S. P. Armes, M. Smallridge and P. McKenna, Macromolecules, 2004, 37, 2395-2403.

52 N. Shoji, M. Ueno and K. Meguro, J. Am. Oil Chem. Soc., 1976, 53, 165-167.

53 A. Dominguez, A. Fernandez, N. Gonzalez, E. Iglesias and L. Montenegro, J. Chem. Educ., 1997, 74, 1227.

54 N. Shoji, M. Ueno and K. Meguro, J. Am. Oil Chem. Soc., 1978, 55, 297-299.

55 M. C. A. Stuart, J. C. van de Pas and J. B. F. N. Engberts, J. Phys. Org. Chem., 2005, 18, 929-934.

56 P. Greenspan and S. D. Fowler, J. Lipid Res., 1985, 26, 781789.

57 A. K. Dutta, K. Kamada and K. Ohta, J. Photochem. Photobiol., A, 1996, 93, 57-64.

58 D. L. Sackett and J. Wolff, Anal. Biochem., 1987, 167, 228234.

59 K. Zhu, R. A. Bressan, P. M. Hasegawa and L. L. Murdock, FEBS Lett., 1996, 390, 271-274.

60 I. Goldstein, J. Biosci., 1983, 5, 65-71.

61 W. Burchard and W. Richtering, Prog. Colloid Polym. Sci., 1989, 80, 151-163.

62 D. Grünwald, M. C. Cardoso, H. Leonhardt and V. Buschmann, Curr. Pharm. Biotechnol., 2005, 6, 381-386.

63 D. Gupta, S. Sabesan and C. F. Brewer, Eur. J. Biochem., 1993, 216, 789-797. 